At the time of writing, ten trainees had attended the on-call shift. All participants completed a pre- and post-session questionnaire. The on-call shift was a useful learning experience (median score 9), and significantly increased perceived preparedness for on-call work from $3 / 10$ to $7 / 10(p<0.001)$. Confidence was significantly improved in seven domains, most markedly in seclusion reviews, prescribing and mental health act tasks.

Conclusion. The psychiatry virtual-on-call programme fills a niche in the training curriculum and is perceived by trainees to be a useful learning experience. The introductory lecture improved confidence in several domains, but not as effectively as the on-call shift. The on-call shift was well received by participants and significantly improved confidence in 7/10 domains. In summary, the virtual-on-call experience improves preparedness for out-of-hours psychiatry work. Follow-up of participants at the end of their psychiatry rotation will ascertain if they felt the programme to be useful during out-of-hours work.

An analysis of lithium requesting across three hospital trusts in the UK: many people are managed with lithium levels below the current nice guidance lower limit

Adrian Heald ${ }^{1 \star}$, Ceri Parfitt ${ }^{2}$, Chris Duff ${ }^{3}$, Jonathan Scargill ${ }^{4}$, Lewis Green ${ }^{5}$ and Anthony Fryer ${ }^{3}$

${ }^{1}$ University of Manchester; ${ }^{2}$ Royal Stoke University Hospital; ${ }^{3}$ Royal Stoke Hospital; ${ }^{4}$ North Manchester General Hospital and ${ }^{5}$ St Helens and Knowsley Hospital

${ }^{*}$ Corresponding author.

doi: 10.1192/bjo.2021.131

Aims. This study examined lithium results and requesting patterns over a 6-year period, and compared these to guidance.

Background. Bipolar disorder is the 4 th most common mental health condition, affecting $\sim 1 \%$ of UK adults. Lithium is an effective treatment for prevention of relapse and hospital admission, and is recommended by NICE as a first-line treatment.

We have previously shown in other areas that laboratory testing patterns are highly variable with sub-optimal conformity to guidance.

Method. Lithium requests received by Clinical Biochemistry Departments at the University Hospitals of North Midlands, Salford Royal Foundation Trust and Pennine Acute Hospitals from 2012-2018 were extracted from Laboratory Information and Management Systems (46,555 requests; 3,371 individuals). We categorised by request source, lithium concentration and re-test intervals.

Result. Many lithium results were outside the NICE therapeutic window $(0.6-0.99 \mathrm{mmol} / \mathrm{L}) ; 49.3 \%$ were below the window and $6.1 \%$ were above the window (median $[\mathrm{Li}]: 0.61 \mathrm{mmol} / \mathrm{L}$ ). A small percentage were found at the extremes $(3.2 \%$ at $<0.1 \mathrm{mmol} / \mathrm{L}$, $1.0 \%$ at $>1.4 \mathrm{mmol} / \mathrm{L})$. Findings were comparable across all sites.

For requesting interval, there was a distinct peak at 12 weeks, consistent with guidance for those stabilised on lithium therapy. There was no peak evident at 6 months, as recommended for those $<65$ years old on unchanging therapy. There was a peak at 0-7 days, reflecting those requiring closer monitoring (e.g. treatment initiation or results suggesting toxicity).

However, $77.6 \%$ of tests were requested outside expected testing frequencies.

Conclusion. We showed: (a) lithium levels are often maintained at the lower end of the NICE recommended therapeutic range (and the BNF range: $0.4-1.0 \mathrm{mmol} / \mathrm{L}$ ); (b) patterns of lithium results and testing frequency are comparable across three sites with differing models of care; (c) re-test intervals demonstrate a noticeable peak at the recommended 3-monthly interval, but not at 6-monthly intervals; (d) Many tests were repeated outside these expected frequencies (contrary to NICE guidance).

\section{We can check serum lithium levels less often without compromising patient safety: evidence from a multi-centre study}

Adrian Heald ${ }^{1 \star}$, David Holland ${ }^{2}$, Mark Davies ${ }^{3}$, Chris Duff ${ }^{4}$, Ceri Parfitt ${ }^{4}$, Lewis Green ${ }^{5}$, Jonathan Scargill ${ }^{6}$, David Taylor ${ }^{7}$ and Anthony Fryer ${ }^{8}$

${ }^{1}$ University of Manchester; ${ }^{2}$ The Benchmarking Partnership; Mike Stedman, Res Consortium; ${ }^{3}$ Res Consortium; ${ }^{4}$ University Hospitals of North Midlands NHS Trust; ${ }^{5}$ St. Helens \& Knowsley Teaching Hospitals NHS Trust; ${ }^{6}$ Royal Oldham Hospital, Oldham, Pennine Care NHS Foundation Trust; ${ }^{7}$ Institute of Psychiatry, Psychology and Neuroscience, King's College London and ${ }^{8}$ Keele University ${ }^{\star}$ Corresponding author.

doi: 10.1192/bjo.2021.132

Aims. Lithium was first found to have an acute antimanic effect in 1948 with further corroboration in the early 1950s. It took some time for lithium to become the standard treatment for relapse prevention in bipolar affective disorder. In this study, our aims were to examine the factors associated wtih the likelihood of maintaining lithium levels within the recommended therapeutic range and to look at the stability of lithium levels between blood tests. We examined this relation using clinical laboratory serum lithium test requesting data collected from three large UK centres, where the approach to managing patients with bipolar disorder and ordering lithium testing varied.

Method. 46,555 lithium rest requests in 3,371 individuals over 7 years were included from three UK centres. Using lithium results in four categories $(<0.4 \mathrm{mmol} / \mathrm{L} ; \quad 0.40-0.79 \mathrm{mmol} / \mathrm{L}$; 0.80-0.99 $\mathrm{mmol} / \mathrm{L} ; \geq 1.0 \mathrm{mmol} / \mathrm{L}$ ), we determined the proportion of instances where, on subsequent testing, lithium results remained in the same category or switched category. We then examined the association between testing interval and proportion remaining within target, and the effect of age, duration of lithium therapy and testing history.

Result. For tests within the recommended range (0.40-0.99 $\mathrm{mmol} / \mathrm{L}$ categories), $84.5 \%$ of subsequent tests remained within this range. Overall 3-monthly testing was associated with $90 \%$ of lithium results remaining within range compared with $85 \%$ at 6-monthly intervals. At all test intervals, lithium test result history in the previous 12-months was associated with the proportion of next test results on target (BNF/NICE criteria), with 90\% remaining within range target after 6-months if all tests in the previous 12-months were on target. Age/duration of lithium therapy had no significant effect on lithium level stability. Levels within the $0.80-0.99 \mathrm{mmol} / \mathrm{L}$ category were linked to a higher probability of moving to the $\geq 1.0 \mathrm{mmol} / \mathrm{L}$ category (10\%) than those in the $0.40-0.79$ mmolL group (2\%), irrespective of testing frequency. Thus prior history in relation to stability of lithium level in the previous 12 months is a predictor of future stability of lithium level.

Conclusion. We propose that, for those who achieve 12-months of lithium tests within the $0.40-0.79 \mathrm{mmol} / \mathrm{L}$ range, it would be reasonable to increase the interval between tests to 6 months, irrespective of age, freeing up resource to focus on those less concordant with their lithium monitoring. Where lithium level is 